in four different groups of people: young normal controls (YNCs, mean age 23.18 years, $n=35$ ), old normal controls (ONCs, mean age 73.40 years, $n=26$ ), patients with mild cognitive impairment $(\mathrm{MCl}$, mean age 73.10 years, $n=12$ ), and patients with early Alzheimer disease (EAD, mean age 74.69 years, $n=14$ ). All participants completed both tests, which consisted of eight navigational subtests, such as recalling the route, remembering landmarks, and identifying points on the route from photographs and videos, all in reference to a route taken around a hospital lobby (both real-life and computer-generated).

Declining overall performances in both tests were observed in the progression from YNC to $\mathrm{ONC}$, to $\mathrm{MCl}$, and to EAD. The photo and video location subtests were a useful measure of cognitive decline in both test environments, as was the landmark recall subtest. Each group displayed a significantly lower performance score in the virtual environment than in real-life, but the effects were equal across the board, indicating that the VR test is a good method for characterizing navigational defects.

Original article Cushman LA et al. (2008) Detecting navigational deficits in cognitive aging and Alzheimer disease using virtual reality. Neurology 71: 888-895

\section{Defects in transfer RNA processing cause pontocerebellar hypoplasia}

Results of a multinational genetic study suggest that defects in transfer RNA (tRNA) processing may be responsible for many cases of the neurodegenerative disease pontocerebellar hypoplasia $(\mathrm{PCH})$. $\mathrm{PCH}$ is an autosomal recessive disorder characterized by impaired development of the cerebellum and ventral pons. There are six known subtypes, all of which feature severe neurological deficits and usually cause death in infancy or childhood. Budde et al. identified mutations in tRNAsplicing endonuclease subunits in patients with $\mathrm{PCH} 2$ or $\mathrm{PCH} 4$.

The researchers examined 58 individuals with $\mathrm{PCH} ; 52$ individuals were diagnosed with $\mathrm{PCH} 2$, and three each with $\mathrm{PCH} 1$ and $\mathrm{PCH} 4$. Of the 52 individuals with $\mathrm{PCH} 2,47$ were found to share a point mutation (probably originating from a $17^{\text {th }}$ century founder mutation) in the TSEN54 gene; the mutation results in an
Ala307Ser substitution in TSEN54, one of the four subunits of the tRNA-splicing endonuclease complex. Two other individuals with $\mathrm{PCH} 2$ had mutations in the TSEN2 and TSEN34 subunits of the complex. The three participants with $\mathrm{PCH} 4$ also carried the Ala307Ser substitution in TSEN54. Two of these individuals, with particularly severe disease, also had nonsense mutations (premature stop codons) in the TSEN54 gene, leading to the expression of truncated proteins. The third had a second point mutation in TSEN54, leading to a Ser93Pro substitution.

Expression studies showed that TSEN54 was highly expressed in the pons, and the cerebellar dentate and olivary nuclei during the second trimester of pregnancy. Impaired tRNA processing would adversely affect protein synthesis in the developing brain, with potentially catastrophic consequences.

Original article Budde BS et al. (2008) tRNA splicing endonuclease mutations cause pontocerebellar hypoplasia. Nat Genet 40: 1113-1118

\section{CoX inhibition reduces medulloblastoma proliferation}

Medulloblastoma, the most common pediatric malignant brain tumor, has been shown by a new Swedish study to be highly dependent for growth on the activity of prostaglandin $E_{2}$ $\left(\mathrm{PGE}_{2}\right)$, levels of which are known to be increased in many tumors. Interference with $\mathrm{PGE}_{2}$ synthesis by inhibition of cyclo-oxygenase (COX) enzymes inhibited medulloblastoma proliferation both in vitro and in vivo.

Baryawno et al. examined 39 human medulloblastoma and supratentorial primitive neuroectodermal tumors from children and adults; they found expression of COX2, microsomal prostaglandin $E$ synthase-both enzymes required for $P G E_{2}$ synthesis - and the four prostanoid receptors, $\mathrm{EP}_{1}$ to $\mathrm{EP}_{4}$, in all cases. Experiments with medulloblastoma cell lines showed that addition of exogenous $\mathrm{PGE}_{2}$ or the $\mathrm{EP}_{2}$ receptor agonist butaprost stimulated cell proliferation. Inhibition of $\mathrm{PGE}_{2}$ synthesis by addition of the COX inhibitors diclofenac or celecoxib produced a concentration-dependent decrease in cell viability in nine medulloblastoma cell lines. COX inhibition also inhibited the clonogenic capacity of medulloblastoma cells and induced apoptosis. Inhibition of COX2 